\title{
Novas condutas terapêuticas para o Linfoma não Hodgkin
}

\author{
New therapeutic management for Non-Hodgkin Lymphoma \\ Nuevas conductas terapêuticas para Linfoma No Hodgkin
}

Francieli Carolini Pasqualetto ${ }^{*}$, Lidiane Regi de Mattos Soriano², Nathália Maciel Maniezzo Stuchi².

\section{RESUMO}

Objetivo: Revisar na literatura científica explorando as novas formas de tratamento utilizadas no Linfoma não Hodgkin. Métodos: $O$ trabalho foi realizado por meio do levantamento de dados utilizando-se as plataformas de busca Bireme, Medline, Lilacs e PubMed, utilizando-se os termos Linfoma não Hodgkin, tratamento farmacológico e imunoterapia. Resultados e discussões: $O$ tratamento mais usual é a quimioterapia, a radioterapia é utilizada em casos restritos, em casos mais graves, onde não há resposta aos tratamentos mais tradicionais, pode se fazer uso da imunoterapia, como por exemplo com o anticorpo monoclonal Rituximab, que promove reações imunológicas que levam à apoptose das células tumorais, resultando na melhora do quadro do paciente. Considerações finais: A importância deste trabalho é atualizar o conteúdo sobre a nova conduta terapêutica envolvendo a imunoterapia para tratamento desta neoplasia, dado ao aumento gradativo do número de casos nos últimos 25 anos, principalmente na faixa etária dos 60 . É importante explorar as novas formas de tratamento utilizadas, para que no futuro novas pesquisas sejam realizadas na busca de melhores prognósticos para esta doença.

Palavras-chave: Lúpus Eritematoso Sistêmico, Nefrite Lúpica, Glomerulonefrite.

\begin{abstract}
Objective: To review the scientific literature exploring the new forms of treatment used in non-Hodgkin's lymphoma. Methods: Data collection using the Bireme, Medline, Lilacs and PubMed research platforms uses non-Hodgkin's lymphoma, pharmacological treatment and immunotherapy. Results and discussions: The most common treatment is chemotherapy, radiotherapy is used in restricted cases, in more severe cases, it is not applicable to more traditional treatments, but it can also be used in therapy, such as with the monoclonal antibody Rituximab, which promotes Immune Reactions which lead to apoptosis of tumor cells, in effect in the marrow of the patient. Final Considerations: The Importance of Work is to Contain the Content on the New Therapeutic Conduct Involving Immunotherapy for the Treatment of This neoplasm Given the Progressive Increase in the Number of Cases in the Last 25 Years, Mainly in the Age Group of the 60 Important is that the new forms of treatment used for future research are realistic in the search for better prognosis for this disease.
\end{abstract}

Keywords: Systemic Lupus Erythematosus, Lupus Nephritis, Glomerulonephritis.

\section{RESUMEN}

Objetivo: Revisar en la literatura científica explorando las nuevas formas de tratamiento utilizadas en el Linfoma no Hodgkin. Métodos: El trabajo fue realizado por medio del levantamiento de datos utilizando las plataformas de búsqueda Bireme, Medline, Lilacs y PubMed, utilizando los términos Linfoma no Hodgkin, tratamiento farmacológico e inmunoterapia.

\footnotetext{
${ }^{1}$ Faculdade de Ciências Médicas - Universidade Estadual de Campinas (FCM - UNICAMP)

*E-mail: f.pasqualetto@hotmail.com

${ }^{2}$ Centro universitário Padre Albino (UNIFIPA) Catanduva-SP
} 
Resultados y discusiones: El tratamiento más usual es la quimioterapia, la radioterapia se utiliza en casos restringidos, en casos más graves, donde no hay respuesta a los tratamientos más tradicionales, se puede hacer uso de la inmunoterapia, como por ejemplo con el anticuerpo monoclonal Rituximab, que promueve reacciones inmunológicas que conducen a la apoptosis de las células tumorales, resultando en la mejora del cuadro del paciente. Consideraciones finales: La importancia de este trabajo es actualizar el contenido sobre la nueva conducta terapéutica envolviendo la inmunoterapia para el tratamiento de esta neoplasia, dado el aumento gradual del número de casos en los últimos 25 años, principalmente en el grupo de edad de los 60. Es importante explorar las nuevas formas de tratamiento utilizados, para que en el futuro nuevas investigaciones se realicen en la búsqueda de mejores pronósticos para esta enfermedad.

Palabras claves: Systemic Lupus Erythematosus, Lupus Nefritis, Glomerulonephritis.

\section{INTRODUÇÃO}

\section{Classificação e tratamento dos Linfomas}

O Linfoma de Hodgkin é composto por células linfoides normais que sofrem alterações neoplásicas nos tecidos linfoides, entretanto o Linfoma Não Hodgkin (LNH) é um tipo no qual as alterações geram um processo de malignidade, porém as características iniciais das células são preservadas (HARRIS et al. 1979), este por sua vez, apresenta-se com maior periodicidade de lesões do tipo extra nodal, acometendo pulmão, pele e cavidade oral, especialmente $o$ anel de Waldeyer, tonsilas e base de língua, embora também possa ser observada a lesão nodal (JARES et al. 2012).

Desta forma o envolvimento primário dessa doença é percebido sob a forma de lesões extra nodais, podendo acometer tanto o tecido ósseo, como o tecido mole, já o envolvimento secundário abrange a cavidade oral. Dentre as manifestações orais do $\mathrm{LNH}$, pode-se destacar: lesões em tecido mole, onde é possível observar tumefação firme e difusa, podendo ser encontrada a presença ou não de ulceração (DIAZ et al. 2005 e ROMERO et al. 2006).

O diagnóstico do LNH é feito por intermédio de coleta de material de lesão via biópsia incisional e análise histopatológica, podendo ser identificado sob várias formas histológicas, dependendo do seu grau e da diferenciação celular (NEVILLE et al. 2004 e REGEZZI e SCIUBBA, 2000).

Quanto aos fatores para o surgimento desta doença, sugere-se que possam estar envolvidos os fatores hereditários, ambientais, ocupacionais e dietéticos. Indivíduos acometidos por imunodeficiências hereditárias, como a hipogamaglobulinemia, a imunodeficiência comum variável, a síndrome de WiskottAldrich e a ataxiateleangiectasia na qual os portadores têm até $25 \%$ de risco de desenvolver $\mathrm{LNH}$ (FILIPOVICH et al. 2003). Além desses fatores, alguns agentes infecciosos têm sido implicados na gênese do LNH, incluindo o vírus Epstein-Barr (YOUNG e MURRAY, 2003), o vírus linfotrópico de células T humano tipo 17 HTLV-17 (ARISAWA et al. 2000), herpes vírus tipo 8 (NADOR et al. 1996), vírus da hepatite C (MELE et al. 2003 e VISWANATHA e DOGAN, 2007), vírus simiano 4011 e a bactéria Helicobacter pylori (PARSONNET et al. 1994). A infecção pelo vírus Epstein-Barr (EBV) está correlacionado com as células NK, em um estudo anterior exibiu resultado positivo da hibridação in situ codificada por EBV em amostras tumorais, assim sendo, os níveis pré e pós-tratamento do DNA EBV circulante apresentaram implicações prognósticas significativas para pacientes com células NK (LIM et al. 2016 e LEl et al. 2002).

Os LNH são derivados em sua maioria dos linfócitos B, sendo que apenas $10 \%$ são dos linfócitos $T$ e células NK (Natural Killers Cells). As células NK são originadas no sistema imune inato e são amadurecidas no fígado e na medula óssea, sendo capazes de se proliferar desenvolvendo resposta a antígenos sem sensibilizá-los, já as células $T$ são desenvolvidas no timo e apresentam característica de células $T$ de memória, tendo afinidade na proliferação por antígenos e, além disso, fazem parte do sistema imune adaptativo, podendo adquirir células de memória imunológica (SUN e LANIER, 2011 e CERWENKA e LANIER, 2016). 
As células Natural Killer em pacientes com células tumorais foram expressas em PD-L1, apresentando um potencial terapêutico para o futuro (HAN et al. 2014 e CHEN et al. 2013). Estas possuem mecanismos de escape das células tumorais e estão em constante desenvolvimento no câncer, o receptor 1 de morte celular (PD-1) e o seu ligante PD 1 (PD-L1) são moléculas importantes no controle imunológico que estão envolvidas na resposta imune mediadas por células $T$ e são importantes reguladores do processo de carcinogênese, sendo a chave principal dos mecanismos de escape das células tumorais (AHMADZADEH et al. 2009 e DONG et al. 2002).

Em relação às alternativas de tratamentos, na forma agressiva do $\mathrm{LNH}$ é realizado geralmente o tratamento com quimioterápico e recomenda-se a utilização do CHOP (Ciclofosfamida, Doxorrubicina, Vincristina e Prednisona) (COLLEONI e INAOKA, 2007). Porém no LNH de crescimento lento, utiliza-se o tratamento com quimioterápico associado à radioterapia e caso a resposta do paciente não seja satisfatória e as manifestações dos sintomas da doença se agravarem, pode ser incluído como alternativa o tratamento com a imunoterapia. Já quando a manifestação da doença apresenta riscos eminentes que possam comprometer as regiões vitais do organismo, recorre-se a procedimentos cirúrgicos e ao transplante de medula óssea (ANSELL e ARMITAGE, 2005).

\section{Imunoterapia como conduta terapêutica do LNH}

O tratamento para o LNH é executado com quimioterapia, radioterapia e imunoterapia, com o objetivo de aumentar a produção das células de defesa do organismo, eliminando o tumor e a produção de células cancerígenas. Essa patologia, normalmente, responde à maioria das modalidades de tratamento. Sendo assim há algumas variações na escolha dos agentes utilizados para o tratamento do LNH, como a duração e dose a ser utilizada (ANSELL e ARMITAGE, 2005 e FISHER e FISCHER, 2004).

A quimioterapia é geralmente composta da associação de duas ou mais drogas, sob várias formas de administração, pode ser paliativa (indicada apenas para aliviar sinais e sintomas e manter a qualidade de vida do paciente até a sua morte), ou curativa (mais agressiva, com a finalidade de remissão e cura da doença), estas formas variam conforme a idade e risco oferecido ao paciente. Os esquemas quimioterápicos utilizados também dependem do tipo histológico e estadiamento do LNH (FISHER e FISCHER, 2004).

O desenvolvimento dos anticorpos contra as células B, foi realizado em 1985, e somente em 1987 foi realizado em quatro pacientes a administração medicamentosa contendo anticorpo anti-CD20 para linfoma de células B malignas (CLARK et al. 1985 e PRESS et al. 1987). Porém em 1997, este anticorpo é nomeado como Rituximabe, tornando-se a quarta terapia de anticorpos aprovados pela FDA (Food and Drug Administration) nos Estados Unidos (WALDMANN, 2003).

Na década de 1990, foi definida como terapia padrão para os Linfomas Não Hodgkin agressivos o CHOP (Ciclofosfamida, Doxorrubicina, Vincristina e Prednisona), porém mais recentemente, a associação de Rituximabe a este esquema demonstrou um benefício adicional ao tratamento (FISHER E FISHER, 2004)

O Rituximabe é um fármaco, mAb (Anticorpo monoclonal) quimérico contra o CD20, que induz através das células $B$ a citotoxidade celular, dependendo de anticorpos e do sistema complemento, estes efeitos estão envolvidos na sinalização celular, na proliferação, na sobrevivência e na apoptose (BEZOMBES et al. 2011).

Em comparação com os quimioterápicos de primeira ou de segunda linhas, o Rituximabe tem promovido uma melhora nos resultados, sendo que a combinação com o CHOP promove uma resposta de $81-97 \%$, melhorando assim a taxa de sobrevivência do paciente (VOSE, 2006; MARCUS et al. 2008; FOSTER et al. 2009).

Este medicamento apresenta o mecanismo de ação capaz de induzir apoptose, causando assim a morte celular das células cancerígenas (BISHOP, 2012). Além disso, atua na destruição das células $\mathrm{B}$, ocorrendo fagocitose e liberação de grânulos citotóxicos no sistema imunológico (SMITH, 2003). 
No caso dos linfomas indolentes os melhores resultados são combinados com a terapia baseada com Rituximabe com quimioterápicos antineoplásicos (BELO e SOTOMAYOR, 2007). Podem ocorrer diversos efeitos adversos ao uso deste medicamento, dentre eles os mais comuns são: eritema no local da injeção, neutropenia, leucopenia, infecções bacterianas, infecções virais, angioedema, náusea, prurido, rash, febre, calafrio, astenia, cefaleia, redução dos níveis de $\operatorname{lgG}$, bronquite, alopecia, trombocitopenia e entre outros (ROCHE, BULA MABTHERA SC).

Em um estudo publicado pela American Society of Clinical Oncology de 2014 foi relatado efeitos adversos graves em $13 \%$ dos pacientes com Rituximabe IV (Intravenoso), e $15 \%\left(375 \mathrm{mg} / \mathrm{m}^{2}\right), 15 \%\left(625 \mathrm{mg} / \mathrm{m}^{2}\right)$ e $8 \%$ $\left(800 \mathrm{mg} / \mathrm{m}^{2}\right)$ dos pacientes tratados com Rituximabe SC (Subcutâneo) nas respectivas doses (SALAR et al. 2014).

Desta forma, o Rituximabe tem se tornado uma alternativa de tratamento para os pacientes de LNH que não respondem mais as terapias convencionais (HESS et al. 2006).

Após o término do tratamento dos LNH é recomendado realizar testes por meio de exame físico, avaliação laboratorial e tomografias periódicas para diagnóstico precoce de eventuais recidivas. Nos LNH agressivos, as recidivas costumam ocorrer nos primeiros dois anos após o término do tratamento, e a detecção precoce é fundamental para a indicação de terapia potencialmente curativa com alta dose de quimioterápicos ou, quando necessário, medidas como o transplante de medula óssea nos pacientes mais jovens. Os pacientes com LNH indolentes podem apresentar padrão de recidivas mais decorrentes e progressiva refratariedade ao tratamento, até evoluírem para uma transformação histológica que resulta em alto grau de malignidade (COLLEONI e INAOKA, 2007).

\section{OBJETIVO}

O objetivo deste trabalho foi revisar os conteúdos sobre os tratamentos do Linfoma Não Hodgkin, enfatizando os novos fármacos e condutas terapêuticas para o melhor prognóstico e resolução e desta neoplasia.

\section{METODOLOGIA}

O trabalho foi realizado por meio do levantamento de dados utilizando-se as plataformas de busca Bireme, Medline, Lilacs e PubMed, utilizando-se os termos Linfoma não Hodgkin, tratamento farmacológico e imunoterapia.

\section{DISCUSSÃO}

Atualmente para o tratamento de câncer se utiliza as combinações quimioterápicas e radioterápicas, sendo a radioterapia voltada apenas para a região local do tumor, desta forma com um papel restrito no $\mathrm{LNH}$, sendo útil apenas na doença localizada ou para tratamento paliativo de sintomas. Com o avanço da medicina surgiram novas vias de tratamento que tornaram melhor a qualidade de vida do paciente, já que o tratamento radioterápico apresenta desempenho limitado em relação aos novos fármacos que estão sendo estudados no momento.

Os registros hospitalares do Brasil têm mostrado que os linfomas se encontram entre os tipos de câncer mais incidentes, dentre eles estão os Linfomas Não Hodgkin. Segundo o Instituto Nacional do Câncer (INCA), a estimativa de 2018 é de 10.180 casos novos de Linfoma Não Hodgkin no Brasil, sendo 5.370 em homens e 4.810 em mulheres. O Pará tem uma taxa estimada de 1,91 casos de LNH para cada 100 mil homens e 1,78 para cada 100 mil mulheres. Segundo o INCA o número de casos dobrou nos últimos 25 anos na faixa 
etária de 60 anos. O mais comum em adultos, é o subtipo $\mathrm{LNH}$ difuso de grandes células $\mathrm{B}$, que atinge por volta $30 \%$ a $40 \%$ dos casos (ARAÚJO et al. 2008).

A América do Norte, a Austrália, a Nova Zelândia e algumas partes da Europa possuem as mais altas taxas desse tipo de câncer. Em geral, o número de novos casos é menor na África, com exceção do linfoma de Burkitt (um subtipo de LNH), que é causado pela infecção do vírus Epstein-Barr (EBV) entre crianças (INCA, 2011). Nos EUA consta que o LNH é o quinto câncer mais diagnosticado com prevalência de $4 \%$ entre todos os cânceres, além disso, é considerada a nona causa mais recorrente de morte por câncer no sexo masculino e no sexo feminino encontra-se na sétima posição (SIEGEL et al. 2016).

Em um estudo francês conduzido pelo GELA (Grupo de Estudos Sobre Linfomas em Adultos) realizado no começo do século XXI por volta dos anos 2000 , foi possível observar a utilização do Rituximabe como tratamento e este apresentou expressivo sucesso na melhora da qualidade de vida dos pacientes. Sendo assim, foram incluídos 399 pacientes tratados em 86 centros franceses. A idade mediana foi de 69 anos e a taxa de resposta completa foi de $52 \%$ no grupo do Rituximabe e $37 \%$ no grupo sem o anticorpo monoclonal. Foi concluído que o tratamento padrão para pacientes idosos com linfoma agressivo e pesquisa de CD20 positiva deve ser a associação de CHOP e Rituximabe por oito ciclos. Em análise de cinco anos de seguimento mediano, as taxas de SLE (sobrevida livre de eventos) e SG (sobrevida global) permaneceram estatisticamente significativas e superiores no grupo de Rituximabe, favorecendo a associação (COIFFER et al. 2002 e FEUGIER et al. 2005). A adição de Rituximabe também está associada à perda da proteína Bcl2 (MOUNIER et al. 2003), que pertence à família Bcl-2 de proteínas reguladoras da apoptose, e está se correlaciona à resistência e à quimioterapia citotóxica in vitro e in vivo (BARRANS et al. 2008 e HILL et al. 1996). Nos estudos mais recentes, é possível observar o desenvolvimento de novos agentes terapêuticos que atuam como alvo em antígenos específicos ou na regulação da resposta imune dos pacientes. Anticorpos como o CD25 (Daclizumab) (JANIK et al. 2015), CD20 (JACENE et al. 2017) (Rituximabe, Tositumomab) ou CD30 (Brentuximabe) (GRAVANIS et al. 2016 e CONNORS et al. 2017) têm apresentado resultados promissores para o tratamento de $\mathrm{LNH}$.

\section{CONCLUSÃO}

Por meio das buscas realizadas é possível concluir que os novos tratamentos para LNH, baseados na utilização da imunoterapia, trouxeram impactos positivos em relação à expectativa de vida e prognóstico dos pacientes portadores desta neoplasia. Desta forma é válido ressaltar a importância da atualização dos profissionais da saúde e ciência sobre as formas de tratamento do $\mathrm{LNH}$, bem como a necessidade de investimentos em busca de novas drogas que possam ser utilizadas ao combate desta doença.

\section{REFERÊNCIAS}

1. AHMADZADEH M, JOHNSON LA, HEEMSKERK B, et al. Tumor antigen-specific CD8 $T$ cells infiltrating the tumor express high levels of PD-1 and are functionally impaired. Blood. 2009; 114:1537-44.

2. ANSELL SM, ARMITAGE J. Non-Hodgkin lymphoma: diagnosis and treatment. Mayo Clinic Proceedings 80 (8):1087-97, 2005.

3. ARAÚJO LHL, VICTORINO APOS, MELO AC, et al. Linfoma não Hodgkin de alto grau: revisão de literatura. Revista Brasileira de Cancerologia 54 (2): 175-183, 2008.

4. ARISAWA K, SODA M, ENDO S, et al. Evaluation of adult T-cell leukemia/ lymphoma incidence and its impact on non-Hodgkin lymphoma incidence in Southwestern Japan. Int J Cancer. 2000;85(3):319- 24.

5. BARRANS SL, CARTER I, OWEN RG, et al. Germinal center phenotype and bcl-2 expression combined with the International Prognostic Revista Brasileira de Cancerologia 2008; 54(2): 175-183 Index improves patient risk stratification in diffuse large Bcell lymphoma. Blood. 2002;99(4):1136-43.

6. BELO C, SOTOMAYOR EM. Monoclonal antibodies for B cell lymphomas: rituximab and beyond. Hematology Am. Soc. Hematol. Educ. Program., p.233-242, 2007. 
7. BEZOMBES C, FOURNIE JJ, Laurent G. Direct effect of rituximab in B-cell-derived lymphoid neoplasias: mechanism, regulation, and perspectives. Mol Cancer Res 2011; 9: 1435-1442.

8. BISHOP MR. Monoclonal antibodies. 2012. Available at: http://www.meds.com/immunotherapy/monoclonal antibodies.html>.

9. CERWENKA A, LANNIER LL. Memória de células assassinas naturais em infecção, inflamação e câncer. Nat Rev Immunol. 2016; 16 (2): 112-23.

10. CHEN BJ, CHAPUY B, OUYANG J, et al. PD-L1 expression is characteristic of a subset of aggressive B-cell lymphomas and virus- associated malignancies. Clin Cancer Res. 2013; 19:3462-73.

11. CLARK EA, SHU G, LEDBETTER JA. Role of the Bp35 cell surface polypeptide in human B-cell activation. Proc Natl Acad Sci USA. 1985; 82(6): 1766-1770.

12. COIFFIER B, LEPAGE E, BRIÉRE J, et al. CHOP chemotherapy plus rituximab compared with CHOP alone in elderly patients with diffuse large-B-cell lymphoma. N Engl J Med. 2002;346(4):235-42.

13. COLLEONI GW, INAOKA BRJ. Linfomas não-Hodgkin: aspectos clínicos, prognósticos e terapêuticos na era do rituximabe. Dialogue científico, 2007.

14. DIAZ MLP, HARO MLL, FLORES AMA. Linfoma no Hodgkin intraósseo reconstruccíon mandibular secundaria: reporte de caso clínico. Revista Odontológica Mexicana. 2005 Dic; 9(4): 197-202.

15. DONG H, STROME SE, SALOMAO DR, et al. Tumor- associated B7-H1 promotes T-cell apoptosis: a potential mechanism of immune evasion. Nat Med. 2002; 8:793-800.

16. FEUGIER P, VAN HOOF A, SEBBAN C, et al. Long-term results of the RCHOP in the treatment of elderly patients with diffuse large B-cell lymphoma: a study by the Groupe d'Etude des Lymphomes de I'Adulte. J Clin Oncol. 2005;23(18):4117-26.

17. FILIPOVICH AH, MATHUR A, KAMAT D, et al. Primary immunodeficiencies: genetic risk factors for lymphoma. Cancer Res. 1992;52 Suppl 19:S5465-7.

18. FISHER SG, FISHER RI. The epidemiology of non-Hodgkin's lymphoma. Oncogene 23: 6524-6534, 2004

19. FOSTER T, MILLER JD, BOYE ME, et al. Economic burden of follicular non-hodgkin's lymphoma. Pharmacoeconomics, v.27, n.8, p.657-679, 2009.

20. HAN L, LIU F, LI R, et al. Role of programmed death ligands in effective T-cell interactions in extranodal natural killer/T-cell lymphoma. Oncol Lett. 2014; 8:1461 9.

21. HARRIS NL, JAFFE ES, DIEBOLD J, et al. World Health Organization classification of neoplastic diseases of the hematopoietic and lymphoid tissues: report of the Clinical Advisory Commitee Meeting - Airlie House, Virginia, November 1997. J Clin Oncol. 1999;17(12):3835-49.

22. HESS G, FLOHR T, KOLBE K, et al. Effect of rituximab on the long-term outcome after high-dose therapy for relapsed B-cell non- Hodgkin's lymphoma. Ann Hematol. 2006; 85(11): 769-779.

23. HILL ME, MACLENNAN KA, CUNNINGHAN DC, et al. Prognostic significance of BCL-2 expression and bcl-2 major breakpoint region

24. JARES P, COLOMER D, CAMPO E. Molecular pathogenesis of mantle cell lymphoma. J Clin Invest 2012; 122(10): 3416-3423.

25. LEI KI, CHAN LY, CHAN WY, et al. Diagnostic and prognostic implications of circulating cell-free Epstein-Barr virus DNA in natural killer/T-cell lymphoma. Clin Cancer Res. 2002; 8:29-34.

26. LIM SH, HYUN SH, KIM HS, et al. Prognostic relevance of pretransplant Deauville score on PET-CT and presence of EBV DNA in patients who underwent autologous stem cell transplantation for ENKTL. Bone Marrow Transplant. 2016; 51:807-12.

27. MARCUS R, IMRIE K, SOLAL-CELIGNY P, et al. Phase III study of R-CVP compared with cyclophosphamide, vincristine, and prednisone alone in patients with previously untreated advanced follicular lymphoma. J. Clin. Oncol., v.26, n.28, p.4579-4586, 2008.

28. MELE A, PULSONI A, BIANCO E, et al. Hepatitis C virus and B-cell non Hodgkin lymphomas: an Italian multicenter case-control study. Blood. 2003;102(3):996-9.

29. MOUNIER N, BRIERE J, GISSELBRECHT C, et al. Rituximab plus CHOP (R-CHOP) overcomes bcl-2associated resistance to chemotherapy in elderly patients with diffuse large B-cell lymphoma (DLBCL). Blood. 2003;101(11):4279-84.

30. NADOR RG, CESARMAN E, CHADBURN A, et al. Primary effusion lymphoma: a distinct clinicopathologic entity associated with the kaposi's sarcomaassociated herpes virus. Blood. 1996;88(2):645-56.

31. NEVILLE BW, DAMM DD, ALLEN CM, et al. Patologia Oral e Maxilo-Facial. 2 ed. Rio de Janeiro: Guanabara Koogan; 2004.

32. PARSANNET J, HANSEN S, RODRIGUEZ L, et al. Helicobacter pylori infection and gastric lymphoma. N Engl J Med. 1994;330(18):1267-71. 
33. PRESS OW, APPELBAUM F, LEDBETTER JA, et al. Monoclonal antibody 1F5 (anti-CD20) serotherapy of human B cell lymphomas. Blood. 1987; 69(2): 584-591.

34. REGEZI JA, SCIUBBA JJ. Patologia Bucal: correlações clínicas patológicas. 3 ed. Rio de Janeiro: Guanabara Koogan; 2000.

35. Roche. Bula MabTheraSC.

36. ROMERO JA, FILLAT ES, BOUTHELIER TU, et al. Linfoma no Hodgkin primario de mandíbula. Rev Esp Patol. 2006; 39(1): 45-8.

37. SALAR A, AVIVI I, BITTNER B, et al. Comparison of Subcutaneous Versus Intravenous Administration of Rituximab As Maintenance Treatment for Follicular Lymphoma: Results From a Two-Stage, Phase IB Study. J Clin Oncol. 2014 Jun 10;32(17):1782-91.

38. SIEGEL RL, MILLER KD, JEMAL A. Cancer statistics, 2016. CA Cancer J Clin 2016; 66: 7-30.

39. SMITH MR. Rituximab (monoclonal anti-CD20 antibody): mechanisms of action and resistance. Oncogene, v.22, n.47, p.7359-7368, 2003.

40. SUN JC, LANIER LL. Desenvolvimento de células NK, homeostase e função: paralelos com células $\mathrm{T}^{\mathrm{CD}} \mathrm{C}^{+}$. Nat Rev Immunol.2011;11 (10): 645-57.

41. VISWANATHA DS, DOGAN A. Hepatitis C virus and lymphoma. J Clin Pathol. 2007;60(12):1378-83.

42. VOSE JM. Personalized immunotherapy for the treatment of non-Hodgkin's lymphoma: a promising approach. Hematol. Oncol., v.24, n.2, p.47-55, 2006.

43. WALDMANN TA. Immunotherapy: past, present and future. Nat Med. 2003; 9(3): 269- 277.

44. YOUNG LS, MURRAY PG. Epstein-barr virus and oncogenesis: from latent genes to tumours. Oncogene. 2003;22(33):5108-21. 